\title{
Sensor-augmented CSII therapy with predictive low-glucose suspend following total pancreatectomy
}

\author{
E S Scott ${ }^{1,2}$, G R Fulcher1,3 and R J Clifton-Bligh ${ }^{1,3,4}$
}

1Department of Endocrinology, Royal North Shore Hospital, Sydney Australia, 2NHMRC Clinical Trials Centre, The University of Sydney, Sydney, Australia, 3University of Sydney, Sydney, Australia, and ${ }^{4}$ Cancer Genetics Laboratory, Hormones \& Cancer Group, Kolling Institute of Medical Research, Royal North Shore Hospital, Sydney, Australia

Correspondence should be addressed to R J Clifton-Bligh Email

jclifton@med.usyd.edu.au

\section{Summary}

Pancreatogenic diabetes is characterised by recurrent severe hypoglycaemia due to changes in both endocrine and exocrine functions. There are no guidelines to manage these individuals. Herein, we describe the post-operative management of two people who developed pancreatogenic diabetes following total pancreatectomy for neuroendocrine malignancy. In both individuals, diabetes was managed using sensor-augmented predictive low-glucose suspend continuous subcutaneous insulin infusion (CSII). We demonstrate the benefit of sensor-augmented CSII in averting hypoglycaemia whilst optimising glycaemic control. Expected rates of severe hypoglycaemia in individuals with pancreatogenic diabetes can be averted with the use of continuous glucose monitoring (CGM) technology, optimising quality of life and reducing the risk of diabetes-related complications.

\section{Learning points:}

- There are no clear guidelines to manage people with pancreatogenic diabetes.

- We describe the use of CGM with predictive low-glucose suspend continuous subcutaneous insulin infusion (CSII) in the management of two individuals post-pancreatectomy.

- Predictive low-glucose suspend technology can achieve excellent glycaemic control whilst avoiding recurrent and severe hypoglycaemia in people with pancreatogenic diabetes.

- Predictive low-glucose suspend CGM should be considered as an effective therapeutic option for the management of pancreatogenic diabetes.

\section{Background}

Pancreatogenic diabetes is caused by pancreatic pathology, including benign and metastatic disease, pancreatitis or resection of the pancreas (1). The incidence of diabetes following pancreatic surgery varies with the extent of resection; however, it accounts for approximately $8 \%$ of all cases of pancreatogenic diabetes (2). Following total pancreatectomy, there is a universal requirement for insulin; however post-distal or pancreaticoduodenectomy, a new diabetes diagnosis has been reported in $31 \%$ and $18 \%$ of the cases, respectively (1). With increased incidence of, and survival following pancreatic surgery, pancreatogenic diabetes will become increasingly prevalent (2).

There are no specific guidelines to manage pancreatogenic diabetes; however, achieving glycaemic control can be challenging. People with pancreatogenic diabetes are classified as 'brittle', due to the loss of 
insulin, glucagon and pancreatic polypeptide. Following pancreatectomy, there is increased peripheral insulin sensitivity due to upregulation of peripheral insulin receptors as a result of insulin deficiency. Concurrently, hepatic insulin receptors are downregulated, leading to lack of insulin-induced suppression of hepatic gluconeogenesis and hyperglycaemia (3). This hepatic resistance may be mediated by a loss of pancreatic polypeptide. In addition, lack of glucagon impairs hypoglycaemia response (4). Susceptibility to hypoglycaemia is further exacerbated with the loss of exocrine pancreatic function, malabsorption and variable glucose absorption post-prandially (3). Hypoglycaemia is frequent post-pancreatectomy, with one study estimating 10 hypoglycaemic events per patient per month and a $40 \%$ overall rate of severe hypoglycaemia (5). Despite difficulties in achieving tight glycaemic control without recurrent hypoglycaemia, people with pancreatogenic diabetes are at risk of microand macrovascular complications (3).

Continuous subcutaneous insulin infusion (CSII) combined with continuous glucose monitoring (CGM) may be an effective tool to manage these individuals. In particular, recent advances with predictive low-glucose management (PLGM) suspend, as in the Medtronic MiniMed 640G, may facilitate hypoglycaemia avoidance. Here, we present the management of two people with pancreatogenic diabetes and the novel role of sensoraugmented CSII therapy in their treatment.

\section{Case presentation}

\section{Case 1}

A 33-year-old man presented with recurrent parathyroiddependent hypercalcaemia. Investigations identified a pancreatic neuroendocrine tumour, and multiple endocrine neoplasia Type 1 (MEN1) was phenotypically and genetically confirmed (MEN1 c.628_631del (p.Thr210Serfs*13). Hypercalcaemia persisted despite bisphosphonates, sandostatin, chemotherapy and peptide receptor radionuclide therapy. Fifteen months later, he proceeded to surgical resection with a total pancreaticoduodenectomy, en bloc spondylectomy, subtotal gastrectomy, right hemicolectomy, left adrenalectomy and removal of hepatic metastases. Following surgery, he developed hypocalcaemia. Postoperatively, his recovery was slow with the diagnosis of pancreatogenic diabetes complicated by parenteral feeding. Labile blood glucose required frequent endocrinology team review and prolonged his hospital admission.

\section{Case 2}

A 64-year-old woman presented with symptoms of reactive hypoglycaemia and was diagnosed with a pancreatic mass on CT. She proceeded to distal pancreatectomy and was diagnosed with a glucagon-producing lowgrade neuroendocrine tumour. Following surgery, her hypoglycaemic symptoms persisted and fasting glucagon remained elevated $(1019 \mathrm{pg} / \mathrm{mL} \quad(40-140))$. Progress gallium DOTA-TATE PET identified DOTA-TATE avid lesions within the anterior pancreas, which proceeded to enlarge despite medical therapy with octreotide. One year later, she underwent a completion pancreatectomy and histology was consistent with alpha cell hyperplasia and islet cell tumour. There was no evidence of metastasis. Management of hyperglycaemia following surgery became the treatment focus.

\section{Investigation}

Summary of initial investigations included in the case presentation, diagnostic work up of pancreatic pathology not the main focus of the case series so not included here.

\section{Treatment}

\section{Case 1}

He was initially managed with an intravenous insulin infusion, and then transitioned to subcutaneous insulin (twice daily glargine supplemented with meal time aspart) with an altered glycaemic target $(8-12 \mathrm{mmol} / \mathrm{L})$ due to fear of hypoglycaemia. Throughout his hospital admission, glycaemic control ranged from 2 to $22.0 \mathrm{mmol} / \mathrm{L}$, with frequent hypoglycaemic episodes. One month postdischarge, he was commenced on CSII and CGM via the Medtronic MiniMed 640G sensor-augmented insulin pump system. The PLGM suspend feature enabled the suspension of the pump infusion when sensor glucose fell below $4.2 \mathrm{mmol} / \mathrm{L}$ (Fig. 1). He attended for a weekly review of CGM and pump adjustment.

\section{Case 2}

Post-operatively, she was commenced on subcutaneous insulin (bd glargine and aspart) within $24 \mathrm{~h}$ of her surgery, albeit with a higher glycaemic target $(8-12 \mathrm{mmol} / \mathrm{L})$ due to concern regarding hypoglycaemia. Following discharge, glycaemic control was difficult due to recurrent hypoglycaemia and severe gastroparesis. Her diet was predominantly liquid based, and despite 


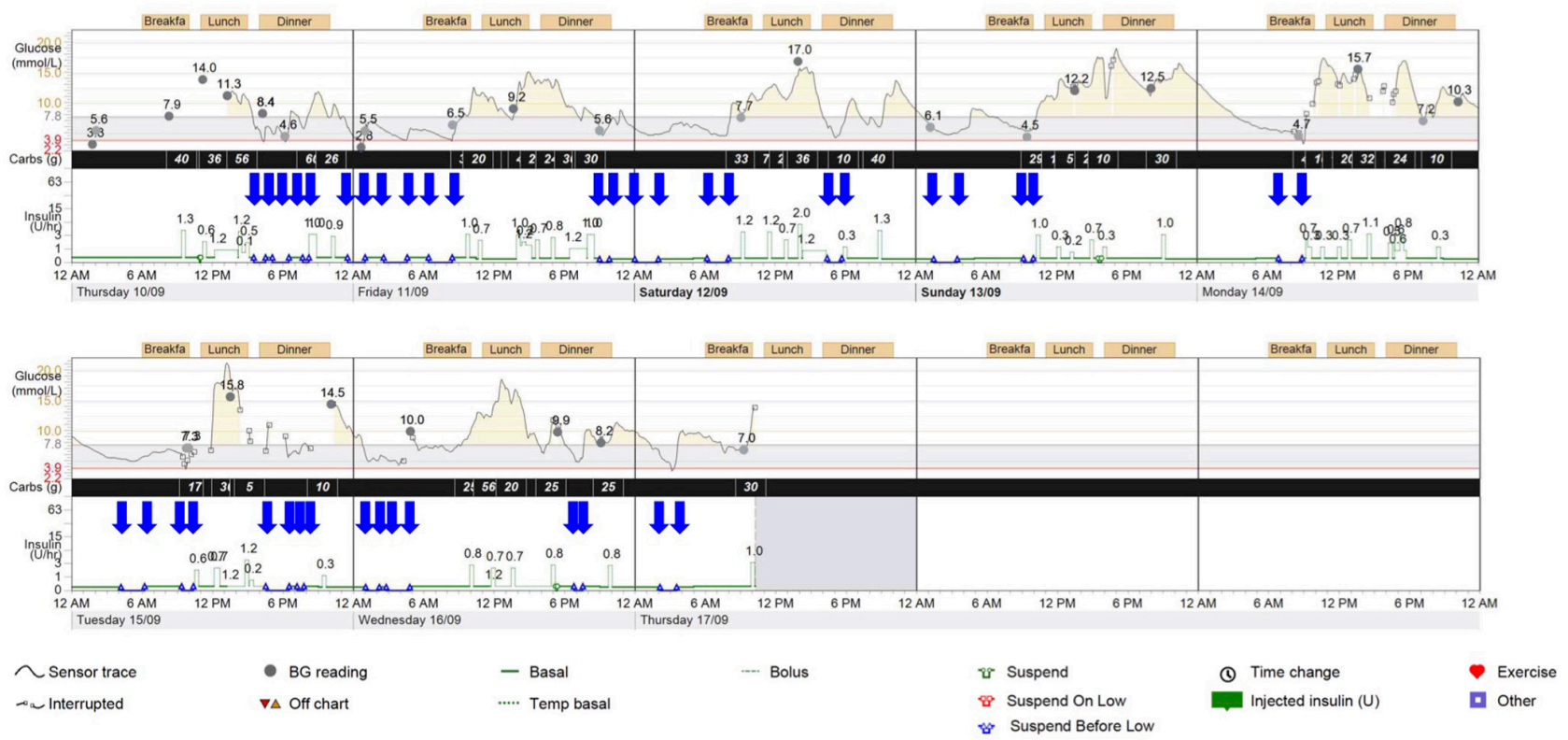

Figure 1

Continuous glucose monitoring (CGM) and metre overview for case 1 dated one month post pancreatectomy. Glucose trace (mmol/L) as detected by CGM is displayed in the top panel, with carbohydrate intake $(\mathrm{g})$ in the middle panel and insulin $(\mathrm{U} / \mathrm{h})$ in the lower panel. Predictive low-glucose management (PLGM) suspend feature enabled pump suspension when blood glucose fell below a pre-set level of $4.2 \mathrm{mmol} / \mathrm{L}$, preventing multiple hypoglycaemic episodes. Pump suspension before hypoglycaemia is indicated by the blue arrow in the bottom panel. The pump suspended over $4 \mathrm{~h}$ and 11 min (3.3 events).

regular domperidone therapy, her symptoms were not controlled. Blood glucose levels ranged between 3 and $18 \mathrm{mmol} / \mathrm{L}$, with daily hypoglycaemia (particularly nocturnal) and an HbA1c of $8.1 \%(65 \mathrm{mmol} / \mathrm{mol})$. She reported severe anxiety that impacted her daily life. 18 months post her completion of pancreatectomy, she was commenced on CSII and CGM via the Medtronic MiniMed 640G sensor-augmented insulin pump. She experienced ongoing gastroparesis and the dual-wave feature was beneficial in matching insulin release to postprandial glucose absorption. Hypoglycaemia was reduced with sensor use, and HbA1c decreased to $7.5 \%$ (59 $\mathrm{mmol} / \mathrm{mol}$ ) (Figure 2).

\section{Outcome and follow-up}

\section{Case 1}

As a result of the sensor-augmented pump, hypoglycaemia was successfully avoided. Eighteen months following surgery, his HbA1c was maintained at $6.2 \%(44 \mathrm{mmol} / \mathrm{mol})$. The patient has experienced only mild and infrequent hypoglycaemia. He is managing his diabetes successfully with the ongoing use of CGM and has been able to return to work and normal activities of daily living.

\section{Case 2}

One year following commencement of the sensoraugmented pump, gastroparesis symptoms and anxiety significantly improved, hypoglycaemia was mild and infrequent and her HbA1c improved to $7 \%(53 \mathrm{mmol} / \mathrm{mol})$.

\section{Discussion}

Closed-loop insulin pumps have been successfully used peri-operatively in individuals undergoing pancreatic resection, achieving glycaemic control without hypoglycaemia (6). A randomised study of 30 people post pancreatic resection demonstrated significantly improved glycaemic control peri-operatively in the closed-loop insulin pump group compared to multiple daily injection, with no difference in hypoglycaemia rates (7). A small study of individuals following total pancreatectomy (including 3 treated by CSII) found that glycaemic control and the incidence of hypoglycaemia did not differ to patients with Type 1 diabetes (4). However, the CSII subset was not analysed individually. To our knowledge, there have been no studies analysing the effect of long-term CSII combined with CGM and PLGM in the pancreatogenic diabetes population. 

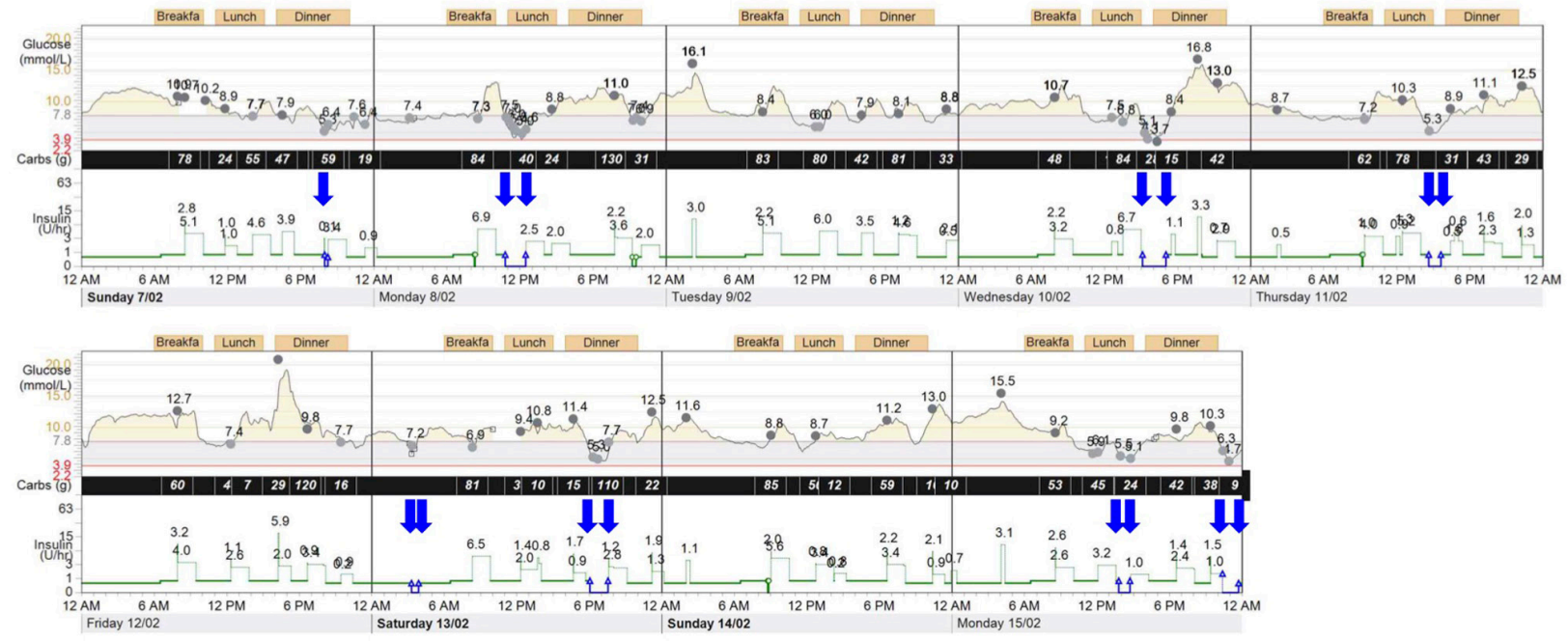

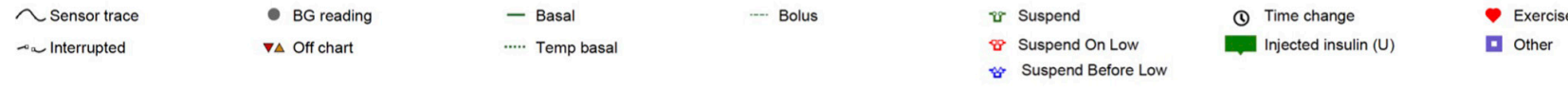

Figure 2

Continuous glucose monitoring (CGM) and metre overview for case 2 dated two months post commencement of continuous insulin infusion therapy. Glucose trace ( $\mathrm{mmol} / \mathrm{L}$ ) as detected by CGM is displayed in the top panel, with carbohydrate intake ( $\mathrm{g}$ ) in the middle panel and insulin (U/h) in the lower panel. Predictive low-glucose management (PLGM) suspend feature enabled pump suspension when blood glucose fell below a pre-set level of $4.0 \mathrm{mmol} / \mathrm{L}$, preventing multiple hypoglycaemic episodes. Pump suspension before hypoglycaemia is indicated by the blue arrow in the bottom panel. The pump suspended over $1 \mathrm{~h}$ and $8 \mathrm{~min}(1.1$ events).

Real-time CGM, where the user is not blinded to the glucose sensor results, significantly improves glycaemic control and also reduces the frequency of hypoglycaemia amongst patients with Type 1 diabetes (8). Sensoraugmented CSII therapy with a PLGM suspend feature enables the cessation of insulin when sensor glucose reaches, or is predicted to reach a hypoglycaemic range within $30 \mathrm{~min}$ (9). The therapy is commercially available in the Medtronic MiniMed 640G insulin pump. PLGM CSII can significantly reduce nocturnal, moderate and severe hypoglycaemia, without a corresponding increase in HbA1c (10). PLGM may significantly reduce the need for hypoglycaemia treatment after an intentional insulin bolus.

The use of a low-glucose suspend sensor-augmented CSII, as in our patients, facilitates intensive insulin therapy whilst avoiding the pitfalls of hypoglycaemia. Low-glucose suspend sensor-augmented CSII should be considered as a viable treatment option for people postpancreatectomy.

Declaration of interest

The authors declare that there is no conflict of interest that could be perceived as prejudicing the impartiality of the research reported.

\section{Funding}

This research did not receive any specific grant from any funding agency in the public, commercial or not-for-profit sector.

\section{Author contribution statement}

$\mathrm{R} J \mathrm{~J}$ Clifton-Bligh was the endocrinologist in charge of the patients. E S Scott, G R Fulcher and RJ Clifton-Bligh wrote and critically revised the manuscript.

\section{References}

1 Burkhart RA, Gerber SM, Tholey RM, Lamb KM, Somasundaram A, McIntyre CA, Fradkin EC, Ashok AP, Felte RF \& Mehta JM 2015 Incidence and severity of pancreatogenic diabetes after pancreatic resection. Journal of Gastrointestinal Surgery 19 217-225. (doi:10.1007/ s11605-014-2669-z)

2 Ewald N \& Bretzel RG 2013 Diabetes mellitus secondary to pancreatic diseases (Type 3c) —are we neglecting an important disease? European Journal of Internal Medicine 24 203-206. (doi:10.1016/j. ejim.2012.12.017)

3 Scavini M, Dugnani E, Pasquale V, Liberati D, Aleotti F, Di Terlizzi G, Petrella G, Balzano G \& Piemonti L 2015 Diabetes after pancreatic surgery: novel issues. Current Diabetes Reports 15 1-11. (doi:10.1007/ s11892-014-0569-y)

4 Jamil LH, Chindris AM, Gill KR, Scimeca D, Stauffer JA, Heckman MG, Meek SE, Nguyen JH, Asbun HJ, Raimondo M, et al. 2012 Glycemic control after total pancreatectomy for intraductal papillary mucinous neoplasm: an exploratory study. HepatoPancreato-Biliary Surgery 2012 381328. (doi:10.1155/2012/381328) 
5 Barbier L, Jamal W, Dokmak S, Aussilhou B, Corcos O, Ruszniewski P, Belghiti J \& Sauvanet A 2013 Impact of total pancreatectomy: shortand long-term assessment. Hepato-Pancreato-Biliary 15 882-892. (doi:10.1111/hpb.12054)

6 Hanazaki K, Yatabe T, Kobayashi M, Tsukamoto Y, Kinoshita Y, Munekage M \& Kitagawa H 2013 Perioperative glycemic control using an artificial endocrine pancreas in patients undergoing total pancreatectomy: tight glycemic control may be justified in order to avoid brittle diabetes. Bio-Medical Materials and Engineering 23 109-116. (doi:10.3233/BME-120736)

7 Okabayashi T, Nishimori I, Yamashita K, Sugimoto T, Maeda H, Yatabe T, Kohsaki T, Kobayashi M \& Hanazaki K 2009 Continuous postoperative blood glucose monitoring and control by artificial pancreas in patients having pancreatic resection: a prospective randomized clinical trial. Archives of Surgery 144 933-937. (doi:10.1001/archsurg.2009.176)

8 Battelino T, Phillip M, Bratina N, Nimri R, Oskarsson P \& Bolinder J 2011 Effect of continuous glucose monitoring on hypoglycemia in type 1 diabetes. Diabetes Care 34 795-800. (doi:10.2337/dc10-1989)

9 Choudhary P, Olsen BS, Conget I, Welsh JB, Vorrink L \& Shin JJ 2016 Hypoglycemia prevention and user acceptance of an insulin pump system with predictive low glucose management. Diabetes Technology and Therapeutics 18 288-291. (doi:10.1089/dia.2015.0324)

10 Bergenstal RM, Klonoff DC, Garg SK, Bode BW, Meredith M, Slover RH, Ahmann AJ, Welsh JB, Lee SW \& Kaufman FR 2013 Threshold-based insulin-pump interruption for reduction of hypoglycemia. New England Journal of Medicine 369 224-232. (doi:10.1056/NEJMoa1303576)

Received in final form 8 September 2017

Accepted 13 September 2017 